\title{
Vertical hybrid microcavity based on a polymer layer sandwiched between porous silicon photonic crystals
}

\author{
F. Yu. Sychev ${ }^{1}$ I. E. Razdolski, ${ }^{1}$ T. V. Murzina, ${ }^{1}$ O. A. Aktsipetrov, ${ }^{1}$ T. Trifonov, ${ }^{2}$ and \\ S. Cheylan 3, a) \\ ${ }^{1}$ Department of Physics, Moscow State University, 119992 Moscow, Russia \\ ${ }^{2}$ Departamento de Ingeniería Electrónica, Universidad Politécnica de Cataluña-UPC, \\ 08034 Barcelona, Spain \\ ${ }^{3}$ ICFO-Institut de Ciencies Fotoniques, Mediterranean Technology Park, \\ 08860 Castelldefels (Barcelona), Spain
}

(Received 17 July 2009; accepted 10 September 2009; published online 19 October 2009)

\begin{abstract}
A vertical hybrid microcavity is fabricated by sandwiching a polymer layer between distributed Bragg reflectors (DBRs) composed of porous silicon photonic crystals. The DBRs are made by electrochemical etching of Si and consist of alternating porous Si layers of high and low porosity, the top DBR being a freestanding film. The hybrid microcavity demonstrates a deep microcavity mode placed within a $200 \mathrm{~nm}$ wide photonic band gap, and reveals a many-fold enhancement of the third-order nonlinearity of the microcavity layer. The fabrication technique employed is rather simple, enabling the use of a variety of functional materials as the microcavity spacer. () 2009 American Institute of Physics. [doi:10.1063/1.3245319]
\end{abstract}

One of the most attractive features of microcavity (MC) photonic band gap (PBG) structures is the localization of optical field in the MC layer at the wavelength resonant to the MC mode. At such wavelength, the local optical field strength is $Q$ times enhanced with respect to that of the incident wave, $Q$ being the MC quality factor. If the MC layer is made out of a functional material, this phenomenon enables to enhance drastically all optical field-dependent effects that this material presents, such as linear and nonlinear absorption, ${ }^{1,2}$ optical harmonics generation, ${ }^{3}$ nonlinear magneto-optical effects, ${ }^{4}$ and luminescence. ${ }^{5}$ Hence composite MCs with a functional MC layer are very attractive photonic structures for potential applications in optoelectronic devices.

Up to now, two main technological approaches are considered for the fabrication of functional vertical MCs. The first one is the deposition of the multilayered distributed Bragg reflectors (DBRs) and of the MC layer in a single technological process, ${ }^{4,6}$ while the second one is the infiltration of a porous MC template by functional materials. ${ }^{7-10}$ These approaches have a common disadvantage which relates to the restriction of the set of functional materials which can be employed for infiltration in nanoporous templates or for the deposition of conjugated layers. One more possibility is the fabrication of one-dimensional hybrid MC based on the separate fabrication of the bottom DBR and of the MC layer. So far, using this approach, only thick metal layer such as aluminum or silver can be used as the top mirror in these structures, ${ }^{11,12}$ which leads to a low MC quality factor.

In this paper, the successful fabrication of a compact functional MC is shown, based on the separate composition of porous silicon DBRs, the top one being a freestanding film, and a spin-coated polymer MC layer. Such a technique results in the fabrication of a viable structure exhibiting a wide $\mathrm{PBG}$, containing a narrow $\mathrm{MC}$ mode, which leads to a

\footnotetext{
a) Author to whom correspondence should be addressed. Electronic mail: stephanie.cheylan@icfo.es.
}

many-fold enhancement of the third-order nonlinearity of the polymer MC layer. This Si-compatible technology is rather simple, inexpensive and enables the use of a variety of functional materials such as dyes, chromophores or nanoparticles, which can be immobilized in a polymer-based MC.

Porous silicon DBRs are fabricated by electrochemical etching of a $p$-type $\mathrm{Si}(100)$ wafer with resistivity $0.005 \Omega \mathrm{cm} .{ }^{13}$ The etching results in the formation of pores with the average diameter of about $10 \mathrm{~nm}$ growing in the direction perpendicular to the (100) surface. The area density of pores and the porosity factor can be controlled by the etching parameters. ${ }^{13}$ Temporal modulation of the etching current results in the formation of a multilayer structure composed of layers with different porosities and, as a consequence, with different refractive indexes. The thickness of the layer is determined by the etching time. In our experiments, layers of high refractive index $n_{1}$ (with low porosity and thickness $d_{1}$ ) and of low refractive index $n_{2}$ (with high porosity and thickness $d_{2}$ ) are formed by etching current densities of 50 and $100 \mathrm{~mA} / \mathrm{cm}^{2}$, respectively. Both the bottom and the top porous silicon mirrors consist of 14 layers (seven pairs) with alternating refractive indices $n_{1}$ and $n_{2}$, the optical thickness of $n_{1} d_{1}=n_{2} d_{2}=\lambda_{0} / 4=210 \mathrm{~nm}$, where $\lambda_{0}$ $=840 \mathrm{~nm}$ is the center of the designed PBG. In our studies, Disperse Red 1 (DR1) incorporated at $4.5 \%$ by weight into polymethylmethacrylate (PMMA) has been chosen as the active organic material of the MC layer. ${ }^{14}$ The polymer solution is spin-coated onto the porous substrates to obtain about $240 \mathrm{~nm}$ thick polymer films (measured with a Dektak 6m profilometer). The average deviation of the MC layer thickness is less than $5 \%$. The top mirror is a freestanding PC fabricated by the same etching process, and released from the Si substrate by a pulse of etching current of about $1 \mathrm{~A} / \mathrm{cm}^{2}$. Like the bottom mirror, it consists of 14 layers with the same values of $n_{1}, d_{1}, n_{2}$, and $d_{2}$. In order to deposit the top DBR onto the polymer-coated bottom DBR, both DBRs are immersed in an ethanol bath and are positioned one below the other one. The ethanol solution is then expelled slowly so 


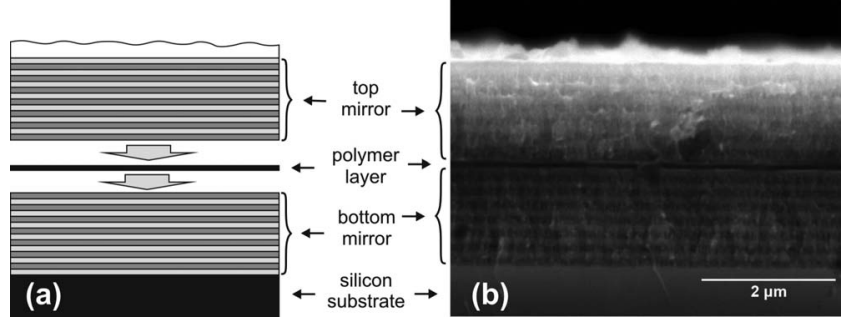

FIG. 1. Schematic of the hybrid DBR/MC/DBR structure (a) and the SEM image of the same structure (b). The dark and bright gray stripes correspond to $p$-Si layers with low and high porosities. The MC layer in the center represents the polymer layer made by spin coating, sandwiched between the two DBR structures.

that the floating mirror ends up resting on the polymer layer with a good mechanical contact. Samples are then kept at a temperature of about $130{ }^{\circ} \mathrm{C}$ for $30 \mathrm{~min}$ in order to remove the solvent, cure the PMMA, and fix the freestanding Bragg mirror onto the polymer layer. A schematic view and a photo of the assembled hybrid MC can be seen in Figs. 1(a) and 2, respectively. As a reference sample, an all-porous-silicon MC is fabricated in a single electrochemical etching process, the parameters of the DBRs and of the porous silicon MC layer being the same as for the hybrid MC structure. Thirdorder nonlinear effects in the hybrid MC are studied applying the reflection $Z$-scan technique, ${ }^{15}$ using a tunable OPO laser system at a wavelength of $750 \mathrm{~nm}$, with a $15 \mathrm{~ns}$ pulsewidth and $70 \mu \mathrm{W}$ average power, the angle of incidence being of about $5^{\circ}$. The reflected radiation at the fundamental frequency was detected by a photodiode and gated electronics.

Figure 1(b) shows a SEM image of the cross-section of the composite MC. One can clearly see the first DBR on the substrate, then the bright MC polymer layer and the second DBR on top of it. The alternating layers of high and low porosity are also visible for each DBR. The bright region on the upper DBR corresponds to a high-porosity layer formed during the application of a high-current-density etching pulse which releases the porous silicon DBR from the silicon substrate. The image shows a very uniform structure with sharp interfaces between the distributed mirrors and the polymer layer, as well as the absence of any inclusions and distortions of the layers. Figure 2 presents a picture of the assembled MC. The adhesion of the freestanding film on top of the polymer spacer is uniform and planar over the whole film surface.

Figure 3 shows the reflection spectra of the hybrid MC and of an all-porous-silicon reference MC. Both MCs have well-pronounced $200 \mathrm{~nm}$ wide PBGs with sharp edges and reflection coefficient within the PGB of above 0.9. The reflectance spectra also present a dip in the stop-band at about $800 \mathrm{~nm}$, in the center of the band gaps. This dip reveals the

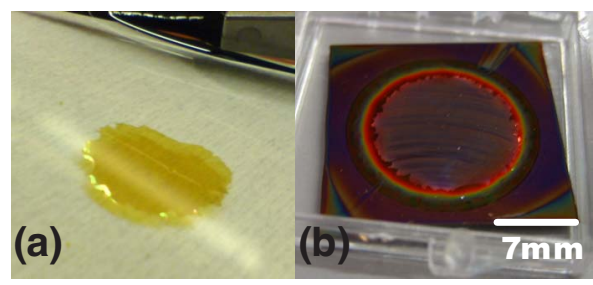

FIG. 2. (Color online) Pictures of a freestanding top mirror (a) and an assembled hybrid MC (b).

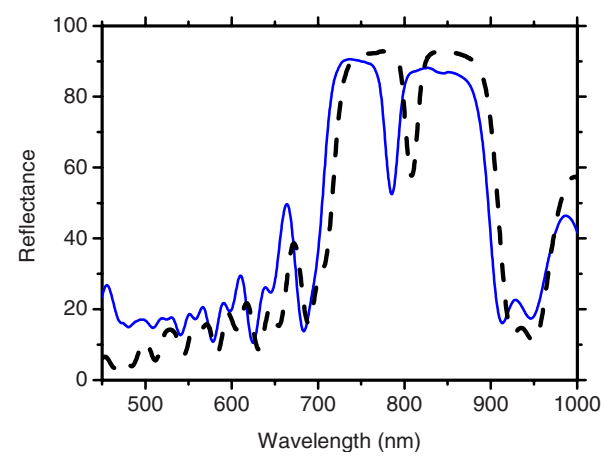

FIG. 3. (Color online) Spectra of the reflectance coefficient for all-poroussilicon MC (dash line) and a hybrid MC (solid line) with similar structural parameters for the top and bottom mirrors.

formation of a resonance MC mode with the quality factor, $Q$, of about 45 . The spectrum of the hybrid $\mathrm{MC}$ is very similar to the spectrum of the all-porous-silicon reference $\mathrm{MC}$, which proves that the optical quality of the hybrid structure is good. In particular, the optical thickness of the polymer MC layer is very close to the one of the reference MC layer. The control of the distance between the two mirrors is a critical issue for vertical MCs as this parameter determines the resonance wavelength of the structure. Moreover, a good homogeneity of the MC layer and a perfect adhesion of the top DBR to the polymer MC layer keep the quality factor of the composite $\mathrm{MC}$ as high as can be achieved by means of the well-developed technique of porous silicon etching and for relatively small number of layers in the DBR, as in the fabricated samples.

To study the photon trapping and the enhancement of the local optical field in the polymer MC spacer, the reflection $Z$-scan $(R Z)$ technique was applied. The scheme of the method is shown on the inset in Fig. 4(a). A $8 \mathrm{~cm}$ focus distance lens, mounted on a translation stage was moved along the beam direction, so that the light intensity on the sample was varied continuously. For the nonlinear refraction measurements a closed-aperture scheme was used, ${ }^{16}$ while the open-aperture $R Z$-scheme was applied for the nonlinear absorption experiments. In both cases, the measured parameter was the normalized reflection coefficient, $R(z)$ $=R^{\prime}(z) / R^{\prime}(\infty)$, where $R^{\prime}(z)$ is the intensity measured for the lens coordinate $z, R^{\prime}(\infty)$ is the intensity when the sample is far from the lens focus, so that the optical power density at

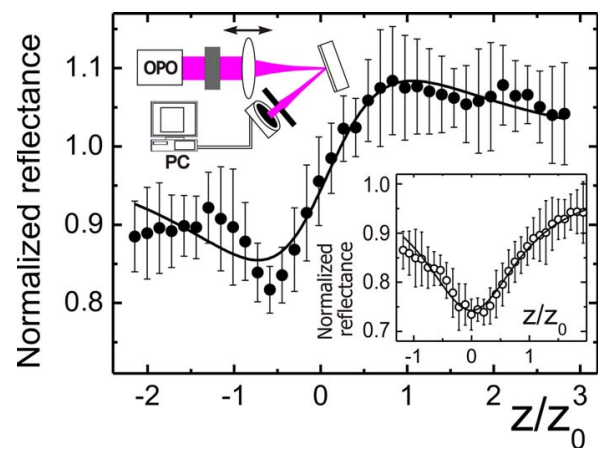

FIG. 4. (Color online) Z-scan of the fabricated hybrid MC (dark circles). The inset in the top left corner is a schematic of the experiment while the bottom right corner inset represents the dependence of the light intensity detected by the open-aperture detector as a function of the distance between the hybrid MC sample and the focus of the lens. 
the MC is small and nonlinear effects can be neglected. In case of the closed-aperture experiments the $R^{\prime}(z)$ dependencies from the hybrid MC were calibrated using those obtained for a single silicon wafer used as a mirror, as its thirdorder nonlinearity is several orders of magnitude smaller compared to DR1-doped PMMA. ${ }^{17}$ This calibration is necessary to exclude intensity variations due to changes in the distance between the detector and the lens.

Figure 4 shows typical $R Z$-scan curves measured for the hybrid MCs, the normalized reflection coefficient being plotted as a function of $z / z_{0}$, where $z_{0}$ is the diffraction lens parameter, which is about $1.4 \mathrm{~cm}$ in our experiments. In both cases, a pronounced light-induced modulation of the refractive index as well as of the absorption coefficient is observed, the maximal changes being attained in the vicinity of the lens focus plane. In the case of the nonlinear refraction, the refractive index can be written as $n=n_{0}+n_{2} I$, where $n_{2} I$ is the light-induced nonlinear contribution, $I$ is the light intensity and $n_{2}$ is the nonlinear refraction index. At the resonant MC wavelength, the $n_{2}$ value deconvoluted from the $R Z$-scan measurement is about $2 \times 10^{-8} \mathrm{~cm}^{2} / \mathrm{W}$, which is at least three orders of magnitude larger than the corresponding nonlinearity of a DR1-doped PMMA film. ${ }^{14,17}$ From the openaperture measurements, the nonlinear absorption index $\beta$ was estimated to be approximately $2 \times 10^{-3} \mathrm{~cm} / \mathrm{W}$, which is also significantly larger than the reported values at lower wavelengths. ${ }^{14,17}$ Large values of the effective third-order nonlinearity observed in the experiments are caused evidently by the light localization and photon trapping in the MC structure. As discussed in, ${ }^{18}$ the enhancement coefficient in a vertical MC can reach the value of $Q^{2}, Q$ being about 45 in our samples. The quadratic dependence on $Q$ arises from two phenomena, namely, because of the local electromagnetic field enhancement in the MC spacer and of a multipass character of the beam propagation inside the structure. The former one affects directly the intensity of the incident beam inside the nonlinear material, while the latter increases the effective light path.

In conclusion, a vertical hybrid MC is fabricated by inserting a polymer layer between two porous silicon DBRs, the upper one being a freestanding porous silicon DBR. A thin polymer film spin-coated on top of the bottom DBR is used as the half-wavelength thick MC spacer. SEM study of the $\mathrm{MC}$ cross section shows an excellent structural quality of the fabricated composite MC. Optical spectra also exhibit good quality PBG and a high $Q$ factor of the hybrid MC.
Many-fold enhancement of the third-order nonlinearity of the polymer within the hybrid structure reveals a strong enhancement of the optical field due to the photon trapping in the MC layer. The suggested technique offers a good perspective for the fabrication of functional PBG MCs using an organic spacer being deposited by spin-coating. The polymer can actually be used as a matrix for a broad set of functional materials, e.g., dyes or nanoparticles.

This work was carried out with the financial support from the Russian Foundation for Basic Research Grant 0902-92435, NATO Collaborative Linkage Grant No. CBP.NR.CLG 982026, and the Consolider-Ingenio Grant No. CSD2007-00007. S.C. and T.T. also acknowledge support from the Spanish Ministry of Education and Science through the Ramon y Cajal and the Juan de la Cierva program, respectively.

${ }^{1}$ R. Herda, T. Hakulinen, S. Suomalainen, and O. G. Okhotnikov, Appl. Phys. Lett. 87, 211105 (2005).

${ }^{2}$ J. M. Lupton, R. Koeppe, J. G. Müller, J. Feldmann, U. Scherf, and U. Lemmer, Adv. Mater. 15, 1471 (2003)

${ }^{3}$ H. Cao, D. B. Hall, J. M. Torkelson, and C.-Q. Cao, Appl. Phys. Lett. 76, 538 (2000).

${ }^{4}$ M. Inoue, R. Fujikawa, A. Baryshev, A. Khanikaev, P. B. Lim, H. Uchida, O. Aktsipetrov, A. Fedyanin, T. Murzina, and A. Granovsky, J. Phys. D: Appl. Phys. 39, R151 (2006).

${ }^{5}$ L. Martiradonna, L. Carbone, M. De Giorgi, L. Manna, G. Gigli, R. Cingolani, and M. De Vittorio, Appl. Phys. Lett. 88, 181108 (2006).

${ }^{6}$ S. I. Khartsev and A. M. Grishin, J. Appl. Phys. 101, 053906 (2007).

${ }^{7}$ T. V. Murzina, F. Yu. Sychev, I. A. Kolmychek, and O. A. Aktsipetrov, Appl. Phys. Lett. 90, 161120 (2007).

${ }^{8}$ F. Jin, C.-F. Li, X.-Z. Dong, W.-Q. Chen, and X.-M. Duan, Appl. Phys. Lett. 89, 241101 (2006).

${ }^{9}$ M. Theander, T. Granlund, D. M. Johanson, A. Ruseckas, V. Sundström, M. R. Andersson, and O. Inganäs, Adv. Mater. 13, 323 (2001).

${ }^{10}$ P. D. García, A. Blanco, A. Shavel, N. Gaponik, A. Eychmüller, B. Rodríguez, L. Liz, and C. López, Adv. Mater. 18, 2768 (2006).

${ }^{11}$ Z. Deng, Y. Zhan, H. Duan, Z. Xiong, F. Bai, and Y. Wang, Synth. Met. 129, 299 (2002).

${ }^{12}$ R. J. Holmes and S. R. Forrest, Phys. Rev. Lett. 93, 186404 (2004).

${ }^{13}$ R. L. Smith and S. D. Collins, J. Appl. Phys. 71, R1 (1992).

${ }^{14}$ L. Brzozowski and E. H. Sargent, J. Mater. Sci. Mater. Electron. 12, 483 (2001)

${ }^{15}$ D. V. Petrov, A. S. L. Gomes, and C. B. de Araujo, Appl. Phys. Lett. 65, 1067 (1994)

${ }^{16}$ M. Sheik-Bahae, A. A. Said, and E. W. Van Stryland, Opt. Lett. 14, 955 (1989).

${ }^{17}$ S. Yamakawa, K. Hamashima, T. Knoshita, and K. Kasaki, Appl. Phys. Lett. 72, 1562 (1998).

${ }^{18}$ I. Razdolski, R. Kapra, T. Murzina, O. Aktsipetrov, and M. Inoue, JETP 84, 451 (2006). 